\title{
Inverse Scattering Transform for the Time Dependent Schrödinger Equation with Applications to the KPI Equation
}

\author{
Xin Zhou
}

Department of Mathematics, University of Wisconsin, Madison, WI 53706, USA

\begin{abstract}
For the direct-inverse scattering transform of the time dependent Schrödinger equation, rigorous results are obtained based on an operatortriangular-factorization approach. By viewing the equation as a first order operator equation, similar results as for the first order $n \times n$ matrix system are obtained. The nonlocal Riemann-Hilbert problem for inverse scattering is shown to have solution.
\end{abstract}

\section{Introduction}

We study in this paper the direct-inverse scattering problem for the $1+1$ time dependent Schrödinger equation:

$$
i \psi_{y}+\psi_{x x}=-u \psi
$$

for real or complex potentials $u$. This problem, besides being of independent physical interest, is connected with the Cauchy problem of the Kadomtsev-Petviashvili (I) (KPI) equation

$$
\left(u_{t}+6 u u_{x}+u_{x x x}\right)_{x}=3 y_{y y} .
$$

It has been formally studied by Zakharov and Manakov [Z-M], [M], Fokas and Ablowitz $[\mathrm{F}-\mathrm{A}]$. For the rigorous theory, certain estimates for the direct scattering transform have been obtained by Segur [S]. The work $[\mathrm{Z}-\mathrm{M}],[\mathrm{M}]$ contains important ideas such as triangular factorization of operators and the derivation of the positivity of the $\bar{\partial}$-scattering data $I+\mathscr{F}$ (see (3.20)) from the unitarity of the physical scattering data $I+\mathscr{S}$ (see (3.5)), while the work [F-A] gives a different approach for deriving $\mathscr{F}$ and constructs for the first time the lump solutions which are two dimensional soliton solutions. However, for the inverse scattering transform, even on the formal level, a satisfactory treatment has not yet been obtained (see $[\mathrm{S}]$ for the comments on [M]).

Our approach is based on viewing Eq. (1.1) as a first order operator equation in $y$ (2.31). For the direct-inverse scattering problem, Eq. (1.1) behaves much more like a first order system than a one dimensional Schrödinger equation 
[G-G-K-M], [D-T]. Comparing it with the first order $n \times n$ system (see [B-C] and $[\mathrm{Z}]$ ), we find many similarities:

1. The triangular factorization of operators takes the place of the triangular factorization of matrices which has played an important role in the theory of the inverse scattering problem for the first order $n \times n$ system.

2. The equations for the inverse problem need to be set up for the solutions normalized on the left or on the right instead of for the analytic solutions (see $[\mathrm{B}-\mathrm{C}]$ and $[\mathrm{Z}]$ ). Thus the scattering data used to build those equations are the triangular factors of $I+\mathscr{F}$ instead of $I+\mathscr{F}$ itself. In fact, the former may be obtained more directly than the latter.

3. The real potentials $u$ give rise to unitary $I+\mathscr{S}$ and positive $I+\mathscr{F}$. A vanishing lemma in this case is available and it implies the invertibility of the inverse equations. The connected time evolution problem (KPI) has no finite time blow-up.

4. For complex potentials, the time evolution exists for a positive time period.

Interestingly enough, the constraint

$$
\int u d x=0,
$$

(or, roughly speaking, that $u$ be in the range of $\partial / \partial x$ ), may be exactly compared to the constraint that the potential be in the range of the derivation ad $J$ in the case of the first order $n \times n$ system. The constraint (1.3) will be made more precise below in (C2.14).

In spite of the similarities enumerated above, the slow decaying property of the lump solutions indicates that the natural inverse scattering problem does not carry the information of arbitrary degree of decay for the potentials $u$. This seems to be the main difference in analytic properties between the first order $n \times n$ system and the $1+1$ time dependent Schrödinger problem. Nontheless, the regularities in both $x$ and $y$ are carried to any order.

In Sect. 2, we introduce certain Banach spaces for the related integral equations. By utilizing the constraint (C2.14), we represent certain solutions of Eq. (1.1) as $I+$ certain Hilbert-Schmidt operator valued functions in $y$. Those solutions are fundamental when they are invertible as operators. The existence of the analytic solution $\psi^{ \pm}$is obtained under the small norm assumption (C2.23), and at this point, the lump solutions are excluded. It is also possible to assume such an existence on the real axis and to add finitely many simple poles (which give rise to the lump solutions) off the real axis as it is done in [F-A]. To solve the direct problem completely (without small norm or genericity assumption) requires further understanding of the nature of the singularities. For instance, we do not know yet whether it is possible for infinitely many poles to exist as in the case of the first order $n \times n$ system (see $[\mathrm{Z}]$ ).

In Sect. 3, we define various scattering data as Hilbert-Schmidt integral operators and derive the relations among them. It seems to be adequate to choose the physical scattering data $\mathscr{S}$ as the "central" scattering data while the others are determined by $\mathscr{S}$ through certain operator triangular factorizations.

In Sect. 4, we set up the needed singular integral equations for the inverse problem which is formulated as a nonlocal Riemann-Hilbert problem. We prove 
a vanishing lemma in the case when $I+\mathscr{S}$ is unitary. This lemma gives the invertibility of the singular integral equations. No small norm assumption is needed. For general $u$, we obtain a weaker result (Proposition 4.19). The time evolution problem of the KPI equation is also briefly discussed.

\section{Integral Equations}

Throughout, we set $\int=\int_{-\infty}^{+\infty}$. We denote by $\psi=\psi(k, x ; y)$ any solutions of $(1.1)$ asymptotical to $(2 \pi)^{-1 / 2} e^{-\infty k^{\infty}-i k^{2} y}$ at $x= \pm \infty$. We also consider an accompanying equation

$$
-i \phi_{y}+\phi_{x x}=-u \phi
$$

and denote by $\phi=\phi(x, k ; y)$ any solutions of $(2.1)$ asymptotical to $(2 \pi)^{-1 / 2} e^{-i k x+i k^{2} y}$ at $x= \pm \infty$. Note that if $u$ is real, Eq. (2.1) is simply the complex conjugate of Eq. (1.1). Below, once any results for Eq. (1.1) are obtained, we expect parallel results for Eq. (2.1). Equation (1.1), (2.1) may be rewritten as

$$
\begin{gathered}
L \mu \stackrel{\text { def }}{=} i \mu_{y}+\mu_{x x}+2 i k \mu_{x}+u \mu=0, \\
\tilde{L} \nu \stackrel{\text { def }}{=}-i v_{y}+v_{x x}-2 i k v_{x}+u v=0,
\end{gathered}
$$

with $\mu \stackrel{\text { def }}{=}(2 \pi)^{1 / 2} e^{-i k x+i k^{2} y} \psi$ and $\nu \stackrel{\text { def }}{=}(2 \pi)^{1 / 2} e^{i k x-i k^{2} y} \phi$. We write $u=u(x ; y), \mu=\mu(k, x ; y)$, and $v=v(x, k ; y)$. Throughout the Fourier transform ' ${ }^{\wedge}$ and the inverse Fourier transform ${ }^{2}$ are between the $x$ and $l$ variables: $\hat{u}=\hat{u}(l ; y), \hat{\mu}=\hat{\mu}(k, l ; y), \check{u}=\breve{u}(l ; y)$, and $\check{v}=\check{v}(l, k ; y)$. It is easier to study the (inverse) Fourier transformed versions of Eqs. (2.2) and (2.3):

$$
\begin{aligned}
i \hat{\mu}_{y}-\left(l^{2}+2 k l\right) \hat{\mu} & =-(2 \pi)^{-1 / 2} \hat{u} * \hat{\mu}, \\
i \check{v}_{y}+\left(l^{2}+2 k l\right) \check{v} & =(2 \pi)^{-1 / 2} \breve{u} * \check{v},
\end{aligned}
$$

where the convolution $*$ is in the $l$ variable. We define the integral operators $g_{k, u}^{l}$, $g_{k, u}^{r}$, and $g_{k, u}^{ \pm}$:

$$
\begin{aligned}
& \left(g_{k, u}^{l} f\right)(l ; y)=i(2 \pi)^{-1 / 2} \int_{-\infty}^{y} d \eta e^{-i l(l+2 k)(y-\eta)} \hat{u} * f(l ; \eta), \\
& \left(g_{k, u}^{r} f\right)(l ; y)=-i(2 \pi)^{-1 / 2} \int_{y}^{+\infty} d \eta e^{-i l(l+2 k)(y-\eta)} \hat{u} * f(l ; \eta), \\
& \left(g_{k, u}^{ \pm} f\right)(l ; y)=i(2 \pi)^{-1 / 2} \int_{ \pm \infty}^{y} d \eta e^{-i l(l+2 k)(y-\eta)} \hat{u} * f(l ; \eta) .
\end{aligned}
$$

We denote by $\mu^{\#}$ the solutions of Eq. (2.2) satisfying

$$
\hat{\mu}^{\#}=(2 \pi)^{1 / 2} \delta+g_{k, u}^{\#} \hat{\mu}^{\#},
$$

where \# denotes $l, r$, or \pm , and $\delta=\delta(l)$ (constant in $y$ ) is the Dirac $\delta$ distribution 
at $l=0$. Let $B$ be a Banach space. A function $f=f(x ; y)$ may be viewed as a $B$-valued function: $f(\cdot ; y) \in B$ for each $y \in \mathbb{R}$;

$$
\|f\|_{\mathbf{L}_{p}(\mathbb{R}, B)} \stackrel{\text { def }}{=}\left(\int\|f(\cdot ; y)\|_{B}^{p} d y\right)^{1 / p}
$$

for $1 \leqq p<\infty$, and

$$
\|f\|_{\mathbf{L}_{\infty}(\mathbb{R}, B)} \stackrel{\text { def }}{=} \operatorname{ess} \sup _{\boldsymbol{y}}\|f(\cdot ; y)\|_{\boldsymbol{B}} .
$$

It is assumed in $[S]$ that $\hat{u} \in \mathbf{L}_{1}\left(\mathbb{R}, \mathbf{L}_{1}(\mathbb{R})\right) \cong \mathbf{L}_{1}\left(\mathbb{R}^{2}\right)$. Clearly, under this assumption, the operator $g_{k, u}^{\#}$ is bounded from $\mathbf{L}_{\infty}\left(\mathbb{R}, \mathbb{C} \delta \oplus \mathbf{L}_{1}(\mathbb{R})\right)$ to $\mathbf{L}_{\infty}\left(\mathbb{R}, \mathbf{L}_{1}(\mathbb{R})\right)$ with their operator norms $\leqq\|\hat{u}\|_{\mathbf{L}_{1}\left(\mathbb{R}^{2}\right)}$. Now let $B$ be a Banach algebra under the convolution and satisfy:

$$
\text { if } f \in \mathbf{L}_{\infty} \text { and } g \in B \text {, then }\|f g\|_{B} \leqq\|f\|_{\mathbf{L}_{\infty}}\|g\|_{B} .
$$

For instance, besides $\mathbf{L}_{1}$, the weighted $\mathbf{L}_{2}$ space $\mathbf{L}_{2}^{\alpha}$ with weight $\alpha>\frac{1}{2}$ is such a Banach algebra under some equivalent norm. We may assume that $\hat{u} \in \mathbf{L}_{1}(\mathbb{R}, B)$. The considered integral operators are then bounded from $\mathbf{L}_{\infty}(\mathbb{R}, \mathbb{C} \delta+B)$ to $\mathbf{L}_{\infty}(\mathbb{R}, B)$. Since $g_{k, u}^{l}$ and $g_{k, u}^{r}$ are Volterra integral operators, $I-g_{k, u}^{l}$ and $I-g_{k, u}^{r}$ are invertible with the estimates:

$$
\left\|\left(g_{k, u}^{l}\right)^{n}\right\|,\left\|\left(g_{k, u}^{r}\right)^{n}\right\| \leqq \frac{\|\hat{u}\|_{\mathbf{L}_{1}(\mathbb{R}, B)}^{n}}{(2 \pi)^{n / 2} n !},
$$

and

$$
\left\|\left(I-g_{k, u}^{l}\right)^{-1}\right\|,\left\|\left(I-g_{k, u}^{r}\right)^{-1}\right\| \leqq \exp \left[(2 \pi)^{-1 / 2}\|\hat{u}\|_{\mathbf{L}_{1}(\mathbb{R}, B)}\right] .
$$

The higher order of regularities in $x$ are contained in the following assumption:

$$
\hat{u} \in \mathbf{L}_{1}\left(\mathbb{R}, \mathbf{L}_{2}^{\alpha}\right) \text { for some integer } \alpha \geqq 1 \text {. }
$$

The solution $\mu=\mu(k, x ; y)$ may also be considered as a function which maps $y$ to $\mu(\cdot, ; y)$. We define the integral operators $\left(g_{u}^{\#} f\right)(k, \cdot ;) \stackrel{\text { def }}{=} g_{k, u}^{\#} f(k, \cdot ;)$.

The parameter $k$ is rather passive so far. However, it will be the space variable in the inverse scattering problem. A successful treatment of the inverse scattering problem therefore requires certain decay in $k$ for the considered data. In the case of $n \times n$ system, since $\operatorname{ran} \operatorname{ad} J=\operatorname{ran}(\operatorname{ad} J)^{j}$ for any positive integer $j$, arbitrary decay in $k$ for the scattering data can be obtained (see [B-C]). However, in our case, only minimum required decay is available through imposing a further assumption on $u$ that

$$
\hat{u} \in \mathbf{L}_{2}\left(|l|^{-1} d l d y\right),
$$

This can also be written as $u \in \operatorname{ran}(\partial / \partial x)^{1 / 2}$. Clearly under this assumption,

$$
\int_{a}^{b} d y e^{i l(l+2 k) y} \hat{u}(l ; y)
$$

for any $-\infty \leqq a \leqq b \leqq+\infty$, defines an $\mathbf{L}_{2}($ dldk) function. We obtain the following two propositions. 
Proposition 2.15. Under conditions (C2.13) and (C2.14), the integral operator $g_{u}^{\#}$ is bounded from $\mathbb{C} \delta+\mathbf{L}_{\infty}\left(\mathbb{R}, \mathbf{L}_{2}\left(\mathbb{R}^{2}\right)\right)$ to $\mathbf{L}_{\infty}\left(\mathbb{R}, \mathbf{L}_{2}\left(\mathbb{R}^{2}\right)\right)$.

Proposition 2.16. $\mu^{l}-1, \mu^{r}-1 \in \mathbf{L}_{\infty}\left(\mathbb{R}, \mathbf{L}_{2}\left(\mathbb{R}^{2}\right)\right)$.

Let $C_{ \pm}: \mathbf{L}_{2}\left(\mathbb{R}^{2}\right) \rightarrow \mathbf{L}_{2}\left(\mathbb{R}^{2}\right)$ be the Cauchy integral operators applied on functions with respect to the first variable:

$$
\left(C_{ \pm} f\right)(k, l)=\frac{1}{2 \pi i} \int \frac{d k^{\prime}}{k^{\prime}-k \mp 0 i} f\left(k^{\prime}, l\right) .
$$

We define $\mathbf{L}_{2}^{ \pm}\left(\mathbb{R}^{2}\right) \stackrel{\text { def }}{=} \operatorname{ker} C_{\mp} \subset \mathbf{L}_{\mathbf{2}}\left(\mathbb{R}^{2}\right)$. If we write

$$
w_{ \pm}(k, l ; y) \stackrel{\text { def }}{=} \int_{ \pm \infty}^{y} d \eta e^{-i l(l+2 k)(y-\eta)} \hat{u}(l ; \eta)
$$

then

$$
w_{ \pm} \in \mathbf{L}_{\infty}\left(\mathbb{R}, \mathbf{L}_{2}^{ \pm}\left(\mathbb{R}^{2}\right)\right)
$$

We assume that

$$
f \in \mathbf{L}_{\infty}\left(\mathbb{R}, \mathbf{L}_{2}^{ \pm}\left(\mathbb{R}^{2}\right)\right) .
$$

Clearly, when $\operatorname{Im} k^{\prime} \neq 0$, the Cauchy integral operator $(2 \pi i)^{-1} \int d k\left(k-k^{\prime}\right)^{-1}$ commutes with the integration in $\left(g_{u}^{\#} f\right)(l, k ; y)$. Therefore if $\pm \operatorname{Im} k^{\prime}<0$,

$$
\frac{1}{2 \pi i} \int d k\left(k-k^{\prime}\right)^{-1}\left(g_{u}^{ \pm} f\right)(l, k ; y)=0 .
$$

Combining (2.18) and (2.19) we conclude:

Proposition 2.21. The operator $g_{u}^{ \pm}$is bounded from $\mathbb{C} \delta+\mathbf{L}_{\infty}\left(\mathbb{R}, \mathbf{L}_{2}^{ \pm}\left(\mathbb{R}^{2}\right)\right)$ to $\mathbf{L}_{\infty}\left(\mathbb{R}, \mathbf{L}_{2}^{ \pm}\left(\mathbb{R}^{2}\right)\right)$.

Proposition 2.22. Under the small norm assumption

$$
\begin{gathered}
\|\hat{u}\|_{\mathbf{L}_{1}\left(\mathbb{R}^{2}\right)}<(2 \pi)^{1 / 2}, \\
\mu^{ \pm}-1 \in \mathbf{L}_{\infty}\left(\mathbb{R}, \mathbf{L}_{2}^{ \pm}\left(\mathbb{R}^{2}\right)\right) .
\end{gathered}
$$

Proof. We write $c=\|\hat{u}\|_{\mathbf{L}_{1}\left(\mathbb{R}^{2}\right)}$. Note that the convolution by and $\mathbf{L}_{1}$ function $f$ is a bounded operator from $\mathbf{L}_{p}$ to $\mathbf{L}_{p}$ with its operator norm $\leqq\|f\|_{\mathbf{L}_{1}}$, we obtain for $j \geqq 1$,

$$
\begin{aligned}
\left\|\left(g_{k, u}^{ \pm}\right)^{j} \delta\right\|_{\mathbf{L}_{2}(\mathbb{R})} & \leqq c^{j-1}\left\|g_{k, u}^{ \pm} \delta\right\|_{\mathbf{L}_{2}(\mathbb{R})} \\
\left\|\left(g_{u}^{ \pm}\right)^{j} \delta\right\|_{\mathbf{L}_{2}\left(\mathbb{R}^{2}\right)} & \leqq c^{j-1}\left\|g_{u}^{ \pm} \delta\right\|_{\mathbf{L}_{2}\left(\mathbb{R}^{2}\right)}
\end{aligned}
$$

Therefore

$$
\hat{\mu}^{ \pm}-(2 \pi)^{1 / 2} \delta=(2 \pi)^{1 / 2} \sum_{j=1}^{\infty}\left(g_{u}^{ \pm}\right)^{j} \delta
$$

converges in $\mathbf{L}_{\infty}\left(\mathbb{R}, \mathbf{L}_{2}^{ \pm}\left(\mathbb{R}^{2}\right)\right)$. 
Noticing the following two facts:

1. The multiplication by $l$ is a derivation with respect to the convolution $*$,

2. By shifting the variable $\eta$ to $\eta+y$ in the integrations in (2.6), (2.7), and (2.8), the $y$-derivatives may be passed to $\hat{u} * f$ as $\eta$-derivatives,

we obtain the following:

Proposition 2.24. If the $y$-derivatives of $\hat{u}$ up to the order $\beta$ are in $\mathbf{L}_{1}\left(\mathbb{R}, \mathbf{L}_{2}^{\alpha}\right) \cap$ $\mathbf{L}_{2}\left(|l|^{-1}\right.$ dldy), then

$$
\frac{\partial^{j+j^{\prime}}}{\partial x^{j} \partial y^{j^{\prime}}}\left(\mu^{l}-1\right), \quad \frac{\partial^{j+j^{\prime}}}{\partial x^{j} \partial y^{j^{\prime}}}\left(\mu^{r}-1\right) \in \mathbf{L}_{\infty}\left(\mathbb{R}, \mathbf{L}_{2}(d k d x)\right) \quad \text { for } \quad j=0, \ldots, \mathrm{a}, j^{\prime}=0, \ldots, \beta,
$$

and under the small norm assumption (C2.23),

$$
\frac{\partial^{j+j^{\prime}}}{\partial x^{j} \partial y^{j^{\prime}}}\left(\mu^{ \pm}-1\right) \in \mathbf{L}_{\infty}\left(\mathbb{R}, \mathbf{L}_{2}^{ \pm}(d k d x)\right) \quad \text { for } j=0, \ldots, \alpha, j^{\prime}=0, \ldots, \beta .
$$

Below, we denote by $\psi$ (respectively $\phi$ the solution corresponding to $\mu^{l}, \mu^{r}$, or $\mu^{ \pm}$ (respectively $v^{l}, v^{r}$, or $v^{ \pm}$), by $\Psi(y)$ (respectively by $\Phi(y)$ ) the integral operator with kernel $\psi(\cdot, ; y)$ (respectively $\phi(\cdot, \cdot ; y))$, and 2.24 by $W(y)$ the unitary multiplier by $e^{-i k^{2} y}$, and by $\boldsymbol{\Psi}_{0}(y)$ the integral operator with the kernel $(2 \pi)^{-1 / 2}(\mu(k, x ; y)-1) e^{i k x}$. Let $\mathbf{F}$ denote the Fourier transform: $\mathbf{L}_{2}(d k) \rightarrow \mathbf{L}_{2}(d x)$. Since $\Psi$ may then be written as

$$
\Psi(y)=W(y)\left(\mathbf{F}^{-1}+\Psi_{0}(y)\right)
$$

it is clear that $\Psi(y)$ is bounded from $\mathbf{L}_{2}(\mathbb{R})$ to $\mathbf{L}_{2}(\mathbb{R})$. Moreover, $\Psi(y)$ is Fredholm with zero index since $\Psi_{0}(y)$ is Hilbert-Schmidt. Similarly we have

$$
\Phi(y)=\left(\mathbf{F}+\Phi_{0}(y)\right) W^{-1}(y),
$$

where $\Phi_{0}(y)$ denotes the integral operator with the $\operatorname{kernel}(2 \pi)^{-1 / 2}(v(x, k ; y)-1) e^{-i k x}$.

Proposition 2.27: $\Psi(y) \Phi(y): \mathbf{L}_{2}(\mathbb{R}) \rightarrow \mathbf{L}_{2}(\mathbb{R})$ is independent of $y$.

Proof. We first assume that $u$ has second order regularity in $y$ as in Proposition 2.24 , then the differentiation of $\psi$ and $\phi$ in $x$ and $y$ shows the boundedness of the following operators:

$$
\begin{gathered}
\Psi(y): \mathbf{H}^{-2} \rightarrow \mathbf{L}_{2}^{-2}, \\
\Phi(y): \mathbf{L}_{2}^{2} \rightarrow \mathbf{H}^{2}, \\
\frac{\partial}{\partial y} \Psi(y): \mathbf{L}_{2} \rightarrow \mathbf{L}_{2}^{-2}, \\
\frac{\partial}{\partial y} \Phi(y): \mathbf{L}_{2}^{2} \rightarrow \mathbf{L}_{2}, \\
\frac{\partial}{\partial y}(\Psi(y) \Phi(y))=\Psi_{y}(y) \Phi(y)+\Psi(y) \Phi_{y}(\mathbf{y}): \mathbf{L}_{2}^{2} \rightarrow \mathbf{L}_{2}^{\dot{-}} .
\end{gathered}
$$

We write Eqs. (1.1), (2.1) into the operator forms: 


$$
\begin{aligned}
i \Psi_{y}(y)+\Psi(y) D^{2}+\Psi(y) U(y) & =0 \\
-i \Phi_{y}(y)+D^{2} \Phi(y)+U(y) \Phi(y) & =0
\end{aligned}
$$

where $D$ denotes the derivative operator, and $U(y)$ denotes the multiplier by $u(\cdot ; y)$. Clearly the left-hand side of (2.33) is a bounded operator from $\mathbf{L}_{2}$ to $\mathbf{L}_{2}^{-2}$ and the left-hand side of (2.34) a bounded operator from $\mathbf{L}_{2}^{2}$ to $\mathbf{L}_{2}$. Substituting (2.33) and (2.34) into (2.32), we obtain that

$$
\frac{\partial}{\partial y}(\Psi \Phi)=0
$$

It then follows from the density of $\mathbf{L}_{2}^{2}$ in $\mathbf{L}_{2}$ that $\Psi \Phi$ is independent of $y$. The result for general $u$ may be obtained by a limiting argument.

\section{Scattering Data}

The connections of different scattering data have been formally derived in [S]. Below, we consider the scattering data defined through both $\mu$ and $v$ with the emphasis of their triangularities. The results in the previous section enable us to interpret these connections through operator operations in $\mathbf{L}_{2}$ space.

We define

$$
\begin{aligned}
& T^{ \pm}(k, k+l)=-i(2 \pi)^{-1} H( \pm l) \int d \eta \hat{u} * \hat{\mu}^{ \pm}(k, l ; \eta) e^{i l(l+2 k) \eta}, \\
& \tilde{T}^{ \pm}(k+l, k)=i(2 \pi)^{-1} H(\mp l) \int d \eta \check{u} * \check{v}^{ \pm}(k, l ; \eta) e^{-i l(l+2 k) \eta}, \\
& R^{ \pm}(k, k+l)=i(2 \pi)^{-1} H(\mp l) \int d \eta \hat{u} * \hat{\mu}^{ \pm}(k, l ; \eta) e^{i l(l+2 k) \eta}, \\
& \tilde{R}^{ \pm}(k+l, k)=-i(2 \pi)^{-1} H( \pm l) \int d \eta \check{u} * \check{\nu}^{ \pm}(k, l ; \eta) e^{-i l(l+2 k) \eta},
\end{aligned}
$$

and

$$
\begin{aligned}
& S(k, k+l)=-i(2 \pi)^{-1} \int d \eta \hat{u} * \hat{\mu}^{r}(k, l ; \eta) e^{i l(l+2 k) \eta}, \\
& \tilde{S}(k+l, k)=-i(2 \pi)^{-1} \int d \eta \check{u} * \check{v}^{r}(k, l ; \eta) e^{-i l(l+2 k) \eta},
\end{aligned}
$$

where $H$ denotes the Heaviside function. We shall show below that the $\bar{\partial}$-scattering data $T^{ \pm}$and $R^{ \pm}$are uniquely determined by the physical data $S$ through operator triangular factorizations. Note that $T^{+}, R^{-}$are upper triangular and $T^{-}, R^{+}$are lower triangular. The singular integral equations for the inverse scattering transform will be built up directly from $T^{ \pm}$and $R^{ \pm}$.

Integration by parts shows

Proposition 3.7. $T^{ \pm}, R^{ \pm}, S, \tilde{T}^{ \pm}, \tilde{R}^{ \pm}, \tilde{S} \in \mathbf{L}_{2}\left(\left(1+(l-k)^{2}\right)^{\alpha}\left(1+\left(l^{2}-k^{2}\right)^{2}\right)^{\beta} d l d k\right)$.

We denote by $\mathscr{T}^{ \pm}, \tilde{\mathscr{T}}^{ \pm}, \mathscr{R}^{ \pm}, \tilde{\mathscr{R}}^{ \pm}, \mathscr{S}$, and $\tilde{\mathscr{S}}$ the integral operators with kernels $T^{ \pm}, \widetilde{T}^{ \pm}, R^{ \pm}, \widetilde{R}^{ \pm}, S$, and $\tilde{S}$ respectively.

Proposition 3.8. $\Psi^{l}, \Psi^{r}, \Psi^{ \pm}, I \pm \mathscr{T}^{ \pm}, \mathscr{I} \pm \mathscr{R}^{ \pm}$, and $I+\mathscr{S}$ are respectively inverses of $\Phi^{l}, \Phi^{r}, \Phi^{ \pm}, I \pm \tilde{\mathscr{T}}^{ \pm}, I \pm \tilde{\mathscr{R}}^{ \pm}$, and $I+\tilde{\mathscr{S}}_{\text {. In addition, we have the following relations }}$ among these operators: 


$$
\begin{aligned}
\Psi^{l} \Phi^{l} & =\Psi^{r} \Phi^{r}=I \\
\Psi^{ \pm} \Phi^{l} & =I \pm \mathscr{T}^{ \pm} \\
\Psi^{l} \Phi^{ \pm} & =I \pm \tilde{\mathscr{T}} \pm \\
\Psi^{ \pm} \Phi^{r} & =I \pm \mathscr{R}^{ \pm} \\
\Psi^{r} \Phi^{ \pm} & =I \pm \tilde{\mathscr{R}}^{ \pm} \\
\Psi^{r} \Phi^{l} & =I+\mathscr{S} \\
\Psi^{l} \Phi^{r} & =I+\tilde{\mathscr{S}}
\end{aligned}
$$

Proof. The identities (3.9)-(3.15) are easily verified by using the fact that

$$
\lim _{y \rightarrow-\infty} \Psi_{0}^{l}(y)=\lim _{y \rightarrow+\infty} \Psi_{0}^{r}(y)=\lim _{y \rightarrow-\infty} \Phi_{0}^{l}(y)=\lim _{y \rightarrow+\infty} \Phi_{0}^{r}(y)=0,
$$

(2.25), (2.26), and Proposition 2.27. From (3.10)-(3.13), we derive

$$
\boldsymbol{\Psi}^{ \pm} \boldsymbol{\Phi}^{ \pm}=\left(I \pm \mathscr{T}^{ \pm}\right)(I \pm \tilde{\mathscr{T}} \pm)=\left(I \pm \mathscr{R}^{ \pm}\right)\left(I \pm \tilde{\mathscr{R}}^{ \pm}\right) .
$$

Since $\mathscr{T}^{ \pm}, \tilde{T}^{ \pm}$are upper triangular and $\mathscr{R}^{ \pm}, \tilde{\mathscr{R}}^{ \pm}$are lower triangular, $(3.16)=I$. The invertibility of $\Psi^{ \pm}$may then be derived from its Fredholm property. Then the remaining part of this proposition follows from the identities we have obtained and the Fredholm property of the considered operators.

The physical scattering data $S$ uniquely determines the $\bar{\partial}$-scattering data $T^{ \pm}$ and $R^{ \pm}$through the triangular factorization

$$
I+\mathscr{S}=\left(I \pm \mathscr{R}^{ \pm}\right)^{-1}\left(I \pm \mathscr{T}^{ \pm}\right)
$$

obtained from (3.10), (3.12), and (3.14).

In the case that $u$ is real, it is straightforward to obtain the following two propositions.

Proposition 3.18. When $u$ is real, the operators $\Psi^{r *}, \Psi^{l *}, \Psi^{ \pm *}, \mathscr{S}^{*}, \pm \mathscr{T}^{ \pm *}$, and $\pm \mathscr{R}^{ \pm *}$ are respectively equal to the operators $\Phi^{r}, \Phi^{l}, \Phi^{\mp}, \tilde{\mathscr{S}}, \pm \tilde{\mathscr{T}}^{\mp}$, and $\mp \tilde{\mathscr{R}}^{\mp}$.

Proposition 3.19. $\Psi^{l}(y), \Psi^{r}(y)$, and $I+\mathscr{S}$ are unitary.

Remark. We shall choose the physical scattering data $S$ to be the "central" scattering data supplemented with the triangular factorizability (3.17). All the results in Proposition 3.18 may be derived from the unitarity of $I+\mathscr{S}$. Also note that the unitarity of the physical scattering data $I+\mathscr{S}$ is equivalent to the positivity of the $\bar{\partial}$-scattering data $I+\mathscr{F} \stackrel{\text { def }}{=}\left(I+\mathscr{T}^{+}\right)\left(I-\tilde{\mathscr{T}}^{-}\right)=\left(I+\mathscr{R}^{+}\right)\left(I-\tilde{\mathscr{R}}^{-}\right)$which relates the analytic solutions $\Psi^{+}$and $\Psi^{-}$:

$$
\Psi^{+}=(I+\mathscr{F}) \Psi^{-} .
$$

To determine $\Psi^{ \pm}$from $\mathscr{F}$ according to (3.20) is called a nonlocal Riemann-Hilbert (factorization) problem. 


\section{Inverse Problem}

We fix both $x$ and $y$, and denote by $\mathscr{A}_{x, y}$ the integral operator with the kernel

$$
A(k, l) e^{i(l-k) x-i\left(l^{2}-k^{2}\right) y} \text {. }
$$

By Proposition 3.7, $\mathscr{F}_{x, y}, \mathscr{T}_{x, y}^{ \pm}, \mathscr{R}_{x, y}^{ \pm}$, etc. are bounded from $\mathbb{C}+\mathbf{L}_{2}$ to $\mathbf{L}_{2}$. The functions $\mu$ and $v$ are then viewed as vectors in $\mathbf{L}_{2}(\mathbb{R})$. The Riemann-Hilbert problem (3.20) may be rewritten as

$$
\mu^{+}=\left(I+\mathscr{F}_{x, y}\right) \mu^{-} .
$$

The vector $\mu^{ \pm}$is said to be a fundamental solution of the Riemann-Hilbert problem (4.1) if the corresponding $\mu^{ \pm}-1 \in \operatorname{ker} C_{\mp}$, and a vanishing solution if $\mu^{ \pm} \in \operatorname{ker} C_{ \pm}$. We may also write the Riemann-Hilbert problem (4.1) into the following different forms:

$$
\begin{aligned}
& \mu^{ \pm}=\left(I \pm \mathscr{T}_{x, y}^{ \pm}\right) \mu^{l}, \\
& \mu^{ \pm}=\left(I \pm \mathscr{R}_{x, y}^{ \pm}\right) \mu^{r} .
\end{aligned}
$$

Proposition 4.4. (Vanishing Lemma). If I $\mathscr{S}$ is unitary, the only vanishing solution of Riemann-Hilbert problem (4.1) is zero.

Proof. Assume that $\mu^{ \pm}$is a vanishing solution of the Riemann-Hilbert problem (4.1). It suffices to show that $\mu^{-}=0$. Since $I+\mathscr{S}$ is unitary, $\left(I-\mathscr{T}_{x, y}^{-}\right)^{-1}=I+\mathscr{T}_{x, y}^{+*}$. Therefore

$$
0=\int d l\left[\overline{\mu^{-}(l, x ; \mathrm{y})} \mu^{+}(l, x ; y)\right]=\|\left(I+\mathscr{T}_{x, y}^{+*}\right) \mu^{-}\left(\cdot, x ; y \|_{\mathbf{L}_{2}}^{2} .\right.
$$

It then follows from the invertibility of $I+\mathscr{T}_{x, y}^{+*}$ that $\mu^{-}(\cdot, x ; y)=0$.

The vanishing lemma is the key to the solvability of the inverse problem in the real potential case. For general potentials, we have the following weaker results,

Proposition 4.6. If we assume the existence of $\Phi^{ \pm}$in (3.11), then the only vanishing solution of the Riemann-Hilbert problem (4.1) is zero.

Proof. Let $\mu^{ \pm}(\cdot, x ; y)$ be a vanishing solution of (4.1). Under the assumption of the Riemann-Hilbert factorizability (3.11), we have

$$
\Phi^{-}(y) \psi^{-}(\cdot, x ; y)=\Phi^{+}(y) \psi^{+}(\cdot, x ; y) .
$$

This may be written explicitly as

$$
\int d l\left[v^{-}\left(x^{\prime}, l ; y\right) \mu^{-}(l, x ; y)\right] e^{i\left(x-x^{\prime}\right) l}=\int d l\left[v^{+}\left(x^{\prime} l ; y\right) \mu^{+}(l, x ; y)\right] e^{i\left(x-x^{\prime}\right) l} .
$$

The left-hand side of (4.7) equals 0 for almost all $x^{\prime} \geqq x$, while the right-hand side equals 0 for almost all $x^{\prime} \leqq x$. It then follows from the invertibility of $\Phi^{ \pm}(y)$ that $\psi^{ \pm}(\cdot, x ; y)=0$.

We study the nonlocal Riemann-Hilbert problem through two integral operators

$$
C_{T_{x, y}} \stackrel{\text { def }}{=} C_{+} \mathscr{T}_{x, y}^{-}+C_{-} \mathscr{T}_{x, y}^{+}
$$




$$
C_{R_{x, y}} \stackrel{\text { def }}{=} C_{+} \mathscr{R}_{x, y}^{-}+C_{-} \mathscr{R}_{x, y}^{+} \text {. }
$$

$C_{T_{x, y}}$ and $C_{R_{x, y}}$ are compact operators from $\mathbb{C}+\mathbf{L}_{2}$ to $\mathbf{L}_{2}$. Using the fact that $\pm C_{ \pm}$are complementary projections, we obtain:

Proposition 4.10. A vector $\mu^{l}$ respectively $\mu^{r}$ is a fundamental solution of the Riemann-Hilbert problem (4.2) respectively (4.3) if and only if it satisfies the equation

$$
\mu^{l}=1+C_{T_{x, y}} \mu^{l},
$$

respectively

$$
\mu^{r}=1+C_{R_{x, y}} \mu^{r} .
$$

Also the homogeneous solutions of these equations are exactly the vanishing solutions of the Riemann-Hilbert problem.

Proposition 4.13. The norm of operator $\left(I-C_{T_{x, y}}\right)^{-1}$ (respectively $\left.\left(I-C_{R_{x, y}}\right)^{-1}\right)$, for a fixed $y_{0}$, is bounded by a number $M_{y_{0}}$ independent of $x$ and $y$ for large $|x|+|y|$ with $y \leqq y_{0}$ (respectively $\left.\geqq y_{0}\right)$.

Proof. From $I \pm \tilde{\mathscr{T}}^{ \pm}=\left(I \pm \mathscr{T}^{ \pm}\right)^{-1}$, we may derive the identity,

$$
\left(I-C_{\tilde{T}_{x, y}}\right)\left(I-C_{T_{x, y}}\right)=I+E_{T_{x, y}},
$$

where

$$
E_{T_{x, y}}=C_{+} \tilde{\mathscr{T}}_{x, y}^{-} C_{-}\left(\mathscr{T}_{x, y}^{+}+\mathscr{T}_{x, y}^{-}\right)+C_{-} \tilde{\mathscr{T}}_{x, y}^{+} C_{+}\left(\mathscr{T}_{x, y}^{+}+\mathscr{T}_{x, y}^{-}\right) .
$$

We proceed to show that the operator $C_{ \pm} \tilde{\mathscr{T}}_{x, y}^{ \pm} C_{\mp}$ vanishes in norm as $|x|+|y| \rightarrow \infty$ with $y \leqq y_{0}$. Consider the $\mathbf{L}_{2}\left(\mathbb{R}^{2}\right)$ rational approximation

$$
\tilde{T}^{ \pm}(k, k+l) e^{-i l(l+2 k) y_{0}} \approx \sum_{i} \frac{a_{i}^{ \pm}(l)}{k-k_{i}}
$$

with $a_{i}^{ \pm}$being smooth functions with compact support in $\mathbb{R}^{ \pm} \backslash 0$.

$$
\begin{aligned}
C_{ \pm} \tilde{\mathscr{T}}_{x, y}^{ \pm} C_{\mp} \phi\left(k^{\prime}\right) \approx & \frac{1}{2 \pi i} \int \frac{d k}{k-k^{\prime} \mp 0 i} \\
& \cdot \int d l \sum_{i} \frac{a_{i}^{\mp}(l) e^{i l x-i l(l+2 k)\left(y-y_{0}\right)}}{k-k_{i}} \frac{1}{2 \pi i} \int \frac{d l^{\prime}}{l^{\prime}-k-l \pm 0 i} \phi\left(l^{\prime}\right) \\
= & \frac{ \pm 1}{2 \pi i} \sum_{\mp \operatorname{lm} k_{\mathrm{t}}>0}\left(k_{i}-k^{\prime}\right)^{-1} \int d l a_{i}^{\mp}(l) e^{i l x-i l\left(l+2 k_{\mathrm{s}}\right)\left(y-y_{0}\right)} \int \frac{d l^{\prime}}{l^{\prime}-l-k_{i}} \phi\left(l^{\prime}\right) .
\end{aligned}
$$

We obtain that $\lim _{y \rightarrow-\infty}\left\|E_{T_{x, y}}\right\|=0$ uniformly in $x$. Using integration by parts, we rewrite (4.17) into the form

$$
\frac{ \pm 1}{2 \pi x} \sum_{\mp \operatorname{lm} k_{i}>0}\left(k_{i}-k^{\prime}\right)^{-1} \int d l^{\prime} \phi\left(l^{\prime}\right) \int d l e^{i l x} \frac{\partial}{\partial l} \frac{a_{i}^{\mp}(l) e^{-i l\left(l+2 k_{i}\right)\left(y-y_{0}\right)}}{l^{\prime}-l-k_{i}} .
$$


Since

$$
\max _{l \in \operatorname{supp} a_{i}^{\mp}}\left|l^{\prime}-l-k_{i}\right|^{-1}
$$

and the square of it are $\mathbf{L}_{2}\left(d l^{\prime}\right)$ functions, for $y$ in a fixed bounded region, the operator $E_{T_{x, y}}$ approaches zero in norms as $|x| \rightarrow \infty$ uniformly in $y$. The proposition then follows from (4.14) and the compactness of $C_{T_{x, y}}$. Similar proof applies to $I-C_{R_{x, y}}$.

For the invertibility of Eqs. (4.11) and (4.12) for every $(x, y)$, when $I+\mathscr{S}$ is unitary, we may use the vanishing lemma and compactness of $\mathscr{T}_{x, y}$ and $\mathscr{R}_{x, y}$ to obtain,

Proposition 4.18. If $I+\mathscr{S}$ is unitary, the operators $I-C_{T_{x, y}}$ and $I-C_{\boldsymbol{R}_{x, y}}$ are invertible for all $x$ and $y$.

For general potentials, we have the following weaker result.

Proposition 4.19. If we assume the factorizability (3.11), then the operators $I-C_{T_{x, y}}$ and $I-C_{R_{x, y}}$ are invertible for all $x$ and $y$.

This proposition says that if the scattering data is obtained from the direct problem, then the equations for the inverse problem are uniquely solvable. Also Proposition 4.19 combined with Proposition 4.13 shows that the Cauchy problem has solution in a positive time period (see the remark following Theorem 4.33).

We denote by $\mathbf{H}^{j, j^{\prime}}$ the $\mathbf{L}_{2}\left(\mathbb{R}^{2}\right)$ Sobolev space smooth to order $j$ in the first variable and smooth to order $j^{\prime}$ in the second variable.

Proposition 4.20. $\left(\partial^{j} / \partial y^{j}\right)\left(\mu^{l}(\cdot, \cdot ; y)-1\right),\left(\partial^{j} / \partial y^{j}\right)\left(\mu^{r}(\cdot, \cdot ; y)-1\right) \in \mathbf{H}^{0, \alpha}$, for $j=0, \ldots, \beta$; and the above derivatives for for $\mu^{l}$ (respectively for $\mu^{r}$ ) approach zero as $y \rightarrow-\infty$ (respectively as $y \rightarrow+\infty$ ).

Proof. The proof will only be given regarding the derivatives of $\mu^{l}$. We have the isometry between $\mathbf{L}_{2}(d l d k)$ and $\mathbf{L}_{2}\left((2|l|)^{-1} d l d(l(l+2 k))\right)$. Thus the Fourier transform

$$
f(k, l)=(2 \pi)^{-1 / 2} \int d \eta \phi(l, \eta) e^{i l(l+2 k) \eta},
$$

gives an isometry between $\mathbf{L}_{2}(d l d k)$ and $\mathbf{L}_{2}\left((2|l|)^{-1} d l d \eta\right)$. We write

$$
T^{-}(k, k+l)=(2 \pi)^{-1 / 2} \int d \eta w^{-}(l, \eta) e^{i l(l+2 k) \eta} .
$$

Then the projection of $\mathscr{T}_{x, y}^{-} 1$ in ran $C_{+}$can be written as

$$
\int_{-\infty}^{0} d l e^{i l x} \int_{-\infty}^{y} d \eta w^{-}(l, \eta) e^{i l(l+2 k)(\eta-y)}
$$

Clearly, as an $\mathbf{L}_{\mathbf{2}}(d k d x)$ function, (4.21) approaches zero as $y \rightarrow-\infty$. Therefore $C_{+} \mathscr{T}_{, y}^{-} 1$ as an $\mathbf{L}_{2}(d k d x)$ function approaches zero as $y \rightarrow-\infty$. Same result may be obtained for $C_{T_{x, y}}$. It then follows from Proposition 4.13 that $\lim _{y \rightarrow-\infty}\left\|\mu^{l}(\cdot, ; y)-1\right\|_{\mathbf{H}^{0, \alpha}}=0$. Similar proofs work for the derivatives.

Let $\psi(k, x ; y)=(2 \pi)^{-1 / 2} \mu(k, x ; y) e^{i k x-i k^{2} y}$, and $\phi(x, k ; y)=(2 \pi)^{-1 / 2} v(x, k ; y) e^{-i k x+i k^{2} y}$. 
Proposition 4.22. Once $\Psi^{l}$ and $\Phi^{l}$ are obtained from the inverse problem, we have

$$
\Phi^{l} \Psi^{l}=\Phi^{+} \Psi^{+}=\Phi^{-} \Psi^{-}=I .
$$

Proof. Since

$$
\Phi^{l} \Psi^{l}=\Phi^{+} \Psi^{+}=\Phi^{-} \Psi^{-}
$$

we have

$$
\int d l\left[v^{-}\left(x^{\prime}, l ; y\right) \mu^{-}(l, x ; y)-1\right] e^{i\left(x-x^{\prime}\right) l}=\int d l\left[v^{+}\left(x^{\prime} l ; y\right) \mu^{+}(l, x ; y)-1\right] e^{i\left(x-x^{\prime}\right) l} .
$$

The left-hand side of (4.24) equals 0 for almost every $\left(x, x^{\prime}\right)$ with $x \leqq x^{\prime}$, while the right-hand side equals 0 for almost every $\left(x, x^{\prime}\right)$ with $x \geqq x^{\prime}$.

Thus by the uniqueness of the Riemann-Hilbert factorization the potential $u$ constructed through $\mu^{l}$ is equal to that constructed from $\mu^{r}$. Once $\mu^{l}$ is solved from Eq. (4.11) for given data $T^{ \pm}$, we let the potential $u$ be an undetermined function in the operator $L$ defined in (2.2). We first assume that $T^{ \pm}$has compact support to obtain,

$$
\begin{aligned}
{\left[C_{T_{x, y}}, L-u\right] \mu^{l} } & =\left[C_{T_{x, y}} L\right] \mu^{l} \\
& =C_{T_{x, y}} L \mu^{l}-L C_{T_{x, y}} \mu^{l} \\
& =\left(C_{T_{x, y}}-I\right) L \mu^{l}+u .
\end{aligned}
$$

If we set

$$
u=\left[C_{T_{x, y}}, L-u\right] \mu^{l},
$$

then $L \mu^{l}=0$ by the injectivity of $I-C_{T_{x, y}}$. The expression (4.26) may be explicitly written as

$$
u(x, y)=\frac{1}{\pi} \frac{\partial}{\partial x} \iint \operatorname{dldk}\left(T^{+}(k, l)+T^{-}(k, l)\right) e^{i(l-k) x-i\left(l^{2}-k^{2}\right) y} \mu^{l}(l, x ; y) .
$$

We split $u$ as:

$$
u=\frac{1}{\pi} \frac{\partial}{\partial x}\left(w_{1}+w_{2}\right)
$$

where

$$
\begin{aligned}
& w_{1}(x ; y)=\iint d l d k\left(T^{+}(k, l)+T^{-}(k, l)\right) e^{i(l-k) x-i\left(l^{2}-k^{2}\right) y} \\
& w_{2}(x ; y)=\iint \operatorname{dldk}\left(T^{+}(k+l, l)+T^{-}(k+l, l)\right) e^{-i k x+i k(k+2 l) y}\left(\mu^{l}(l, x ; y)-1\right) .
\end{aligned}
$$

\section{Proposition 4.28.}

$$
\begin{aligned}
& \left\|\left(\frac{\partial}{\partial x}\right)^{1 / 2} w_{1}\right\|_{H^{\alpha, \beta}} \leqq c_{1}\left\|T^{+}+T^{-}\right\|_{\mathbf{L}_{2}\left(\left(1+(l-k)^{2}\right)^{\alpha}\left(1+\left(l^{2}-k^{2}\right)^{2}\right)^{\beta} d l d k\right)}
\end{aligned}
$$

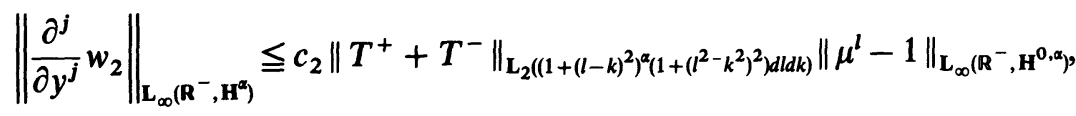

$$
\begin{aligned}
& j=0, \ldots, \beta,
\end{aligned}
$$


and the derivatives in (4.30) approach zero in $\mathbf{H}^{\alpha}$ as $y \rightarrow-\infty$. An estimate similar to (4.30) for $y \in \mathbb{R}^{+}$may be established by using $\mu^{r}$ and $R^{ \pm}$. It is also true that the derivatives approach zero as $y \rightarrow+\infty$.

Proof. The estimate (4.29) follows immediately from the fact that $d(l-k) d\left(l^{2}-k^{2}\right)=$ $2|l-k| d l d k$. To show (4.30), let us first define

$$
v(l, x ; y)=\int d k\left(T^{+}(k+l, l)+T^{-}(k+l, l)\right) e^{-i k x+i k(k+2 l) y} .
$$

Clearly,

$$
\left\|\frac{\partial^{j}}{\partial y^{j}} v\right\|_{\mathbf{L}_{\infty}\left(\mathbb{R}, \mathbf{H}^{0, \alpha}\right)} \leqq c_{3}\left\|T^{+}+T^{-}\right\|_{\mathbf{L}_{2}\left(\left(1+(l-k)^{2}\right)^{\alpha}\left(1^{+}\left(l^{2}-k^{2}\right)^{2}\right)^{j} d l d k\right)} \quad j=0, \ldots, \beta .
$$

Differentiating the formula

$$
w_{2}(x ; y)=\int d l v(l, x ; y)\left(\mu^{l}(l, x ; y)-1\right)
$$

with respect to $x$ and $y$, and then using the Schwartz inequality, we obtain the estimate (4.30). The limit as $y \rightarrow-\infty$ is obtained from the fact that $\lim _{y \rightarrow-\infty} \mu^{l}=1$.

We have shown that under the assumption that $\mathscr{T}^{ \pm}$has compact support, the potential $u$ constructed from (4.27) and $\psi$ satisfy the operator equation (2.33). Every $T^{ \pm}$is a limit of some with compact support. The limiting $u$ and $\psi$ still satisfy Eq. (2.33) and the estimates for $u$ in Proposition 4.28 remains valid because $\mu^{l}$ and $\mu^{r}$ depend continuously on $T^{ \pm}$and $R^{ \pm}$respectively. Unitarity of $I+\mathscr{S}$ implies that $\Phi^{l}=\Psi^{l *}$. This shows,

Proposition 4.32. If $I+\mathscr{S}$ is unitary, the constructed potential $u$ is real.

In the Cauchy problem for the KPI equation, the scattering data evolves as a unitary conjugation: $S_{t}(k, l)=e^{4 i k^{3} t} S_{0}(k, l) e^{-4 i l^{3} t}$, (see any of the mentioned papers about KPI). Thus the triangular factors $T^{ \pm}$and $R^{ \pm}$must evolve in the same way. We obtain the following

Theorem 4.33. If the real initial data $u(\cdot ;, 0)$ satisfies the conditions in Proposition 2.24 and the small norm assumption (C2.23), then the KPI equation (1.2) has a unique solution $u(\because ;, t)$, for all real $t$, with the estimates in Proposition 4.28 , and such $a$ solution is real.

We remark that for nonreal $u(\because ;, 0)$ satisfying the hypotheses in Theorem 4.33, Propositions 4.19, 4.13 together show that the time evolution $u(\cdot ; \cdot, t)$ exists in a neighborhood of $t=0$. Certainly we may also assume that $T^{ \pm}$has small norm to obtain the existence of $u(\cdot ; \cdot, t)$ for all real $t$.

Acknowledgement. A. Nachman has made very useful suggestions to this work.

\section{References}

[B-C]. Beals, R., Coifman, R. R.: Scattering and inverse scattering for first order systems. Commun. Pure Appl. Math. 37, 39-90 (1984) 
[D-T]. Deift, P., Trubowitz, E.: Inverse scattering on the line. Commun. Pure Appl. Math. 32, 121-251 (1979)

[F-A]. Fokas, A. S.: On the inverse scattering of the time-dependent Schrödinger equation and the associated Kadomtsev-Petviashvili (I) equation. Stud. Appl. Math. 69, 211-228 (1983)

[G-G-K-M]. Gardner, C. S., Greene, J. M., Kruskal, M. D., Miura, R. M.: Method for solving the Korteweg-de Vries equation. Phys. Rev. Lett. 19, 1095-1097 (1967)

[M]. Manakov, S. V.: Physica 3D, 420 (1981)

[S]. Segur, H.: Comments on IS for the Kadomtsev-Petviashvili equation, in: Mathematical methods in hydrodynamics and integrability in dynamical systems. AIP Conference Proceedings 88, 211-228 (1982)

[Z-M]. Zakharov, V. E.: Manakov, S. V.: Sov. Sci. Rev. Phys. Rev. 1, 133 (1979)

$[Z]$. Zhou, X.: Direct and inverse scattering transforms with arbitrary spectral singularities. Commun. Pure. Appl. Math. 42, 895-938 (1989)

Communicated by B. Simon

Received July 10, 1989 